\title{
Birgit Grüb
}

\section{Stand und Perspektiven von PPP in der Praxis - zugleich Besprechung einer Grundlagenstudie zu PPP in der Schweiz}

ÖPP-Beschleunigungsgesetz; Organisations-PPP; PPP in der Schweiz; PPP-Rendite; Projekt-PPP; Public Private Partnership

Vor dem Hintergrund der Finanzkrise der Gebietskörperschaften in Deutschland gewinnen Public Private Partnership (PPP) zunehmend an Bedeutung. Sie werden inzwischen für fast alle öffentlichen Aufgabenfelder auf staatlicher und kommunaler Ebene diskutiert und in wesentlichen Bereichen auch umgesetzt. Dabei spielen die Erfahrungen und Ansätze der europäischen Nachbarstaaten eine wesentliche Rolle. Eine Übersicht dieser Entwicklung im europäischen Kontext findet sich in dem Sammelband Bolz, Urs (Hrsg.), Public Private Partnership in der Schweiz, Grundlagenstudie - Ergebnis einer gemeinsamen Initiative von Wirtschaft und Verwaltung.

\section{Aktueller Stand und Grundkategorien von PPP}

Der dem angloamerikanischen entnommene Begriff „Public Private Partnership ${ }^{\text {“1 }}$ steht in der deutschen Diskussion und Praxis für vielschichtige und sehr heterogene Sachverhalte. Gemeinsam ist den mit diesem Begriff erfassten Bereich generell eine Zusammenarbeit und Austauschbeziehung eines öffentlichen und privaten Partners. Trotz aller Heterogenität haben sich inzwischen als Grundkategorien zur Systematisierung und Unterteilung der PPP-Ansätze die auf dem Tauschmodell und dem Poolmodell basierenden Konzepte durchgesetzt. $^{2}$ Sie haben jeweils unterschiedlichen Einfluss auf die Ergebnis-, Vermögens- und Finanzrechnung einer Gebietskörperschaft (vgl. Abb. 1). Im Vordergrund der praktischen Entwicklung stehen auf dem Tauschmodell basierende Projekt-PPP, die sich auf fast alle klassischen Bereiche öffentlicher Aufgabenwahrnehmung erstrecken. ${ }^{3}$ Der Schwerpunkt der praktischen Anwendung liegt allerdings im Infrastrukturbereich und hier insbesondere im Bau-Sektor, ein Sachverhalt, der sich aus dem umfassenden Gutachten PPP im Hochbau erklärt. ${ }^{4}$ Verbunden sind diese Projekt-PPP i.d.R. mit Finanzierungsmodellen und/oder sie sind primär auf die Finanzierung ausgerichtet. Von daher haben PPP-Projekte in den ganz überwiegenden Fällen auch den Charakter von kredit-

Vgl. Budäus/Grüning (1997), S. 25-66.

Diese Kategorisierung kommt auch im EU-Grünbuch (Mai 2004) zum Ausdruck.

Vgl. hierzu anschaulich schon die Untersuchung von Kruzewicz (1993).

Vgl. Beratergruppe - „PPP im öffentlichen Hochbau“ (2003). 
ähnlichen Geschäften. Bei den Organisations-PPP handelt es sich um klassische gemischtwirtschaftliche Unternehmen oder um Finanzbeteiligungen.

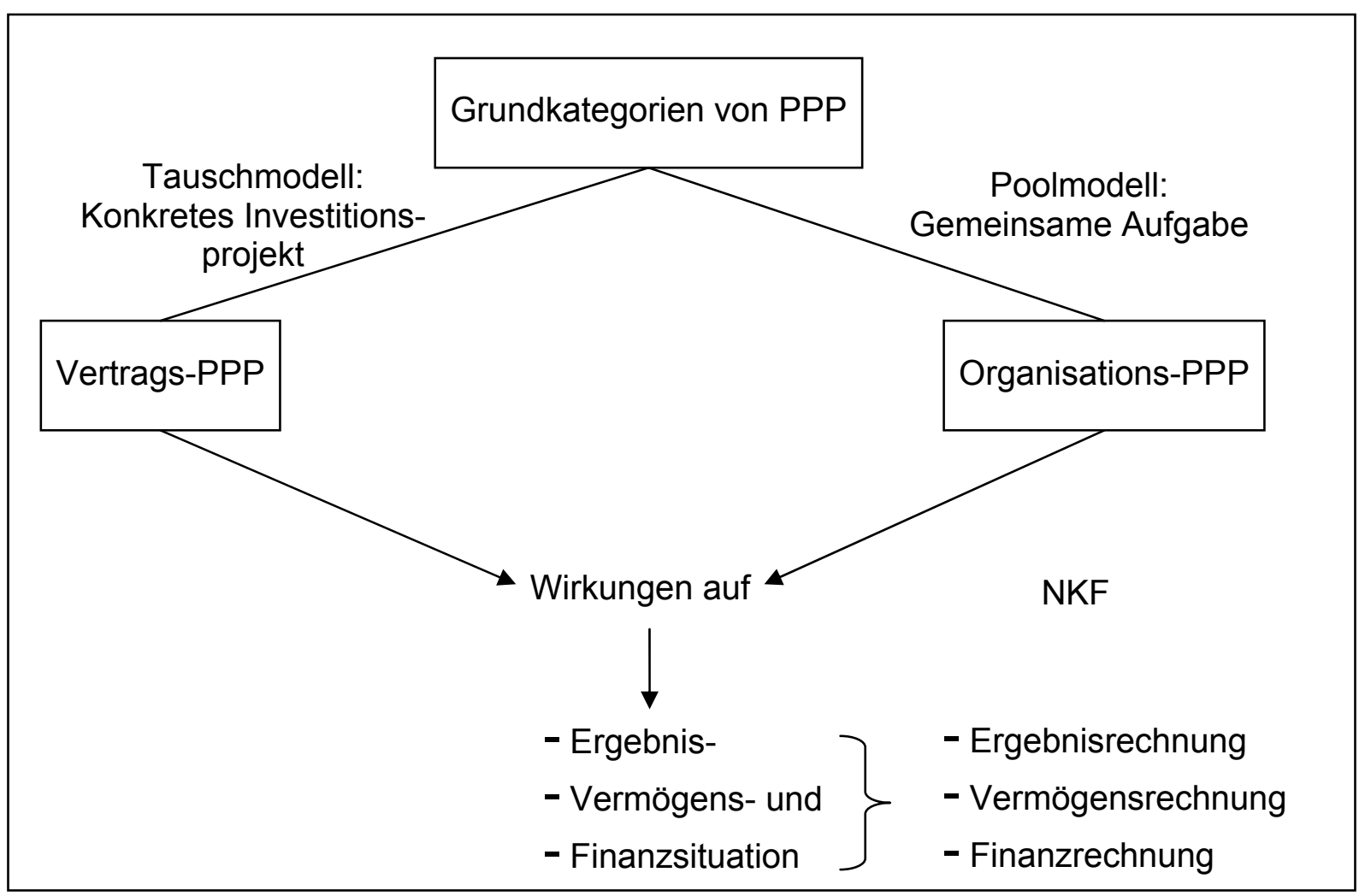

Abb. 1: Grundkategorien von PPP

Quelle: $\quad$ Vgl. Budäus/Grüb (2006)

Ähnlich gestalten sich die in England, einem der Vorreiter der PPP-Entwicklung, eingesetzten Private Finance Initiatives (PFI). Diese stellen eine Kooperation zwischen der öffentlichen Hand und einem privaten Wirtschaftssubjekt dar, bei der das private Unternehmen ein Projekt errichtet, finanziert und über den gesamten Lebenszyklus betreibt. Der Schwerpunkt liegt auch hier auf der Finanzierung. Eingesetzt werden die PFI im Verkehrsbereich, im Gesundheitssektor, aber auch im Bildungssektor. Auf internationaler Ebene zeichnet sich inzwischen ab, dass kaum ein Bereich öffentlicher Aufgabenwahrnehmung existiert, in dem nicht PPP zur Anwendung kommt. ${ }^{5}$ Die Gründe hierfür liegen in der erwarteten höheren Effizienz.

5 Vgl. Hodge/Greve (2005). 


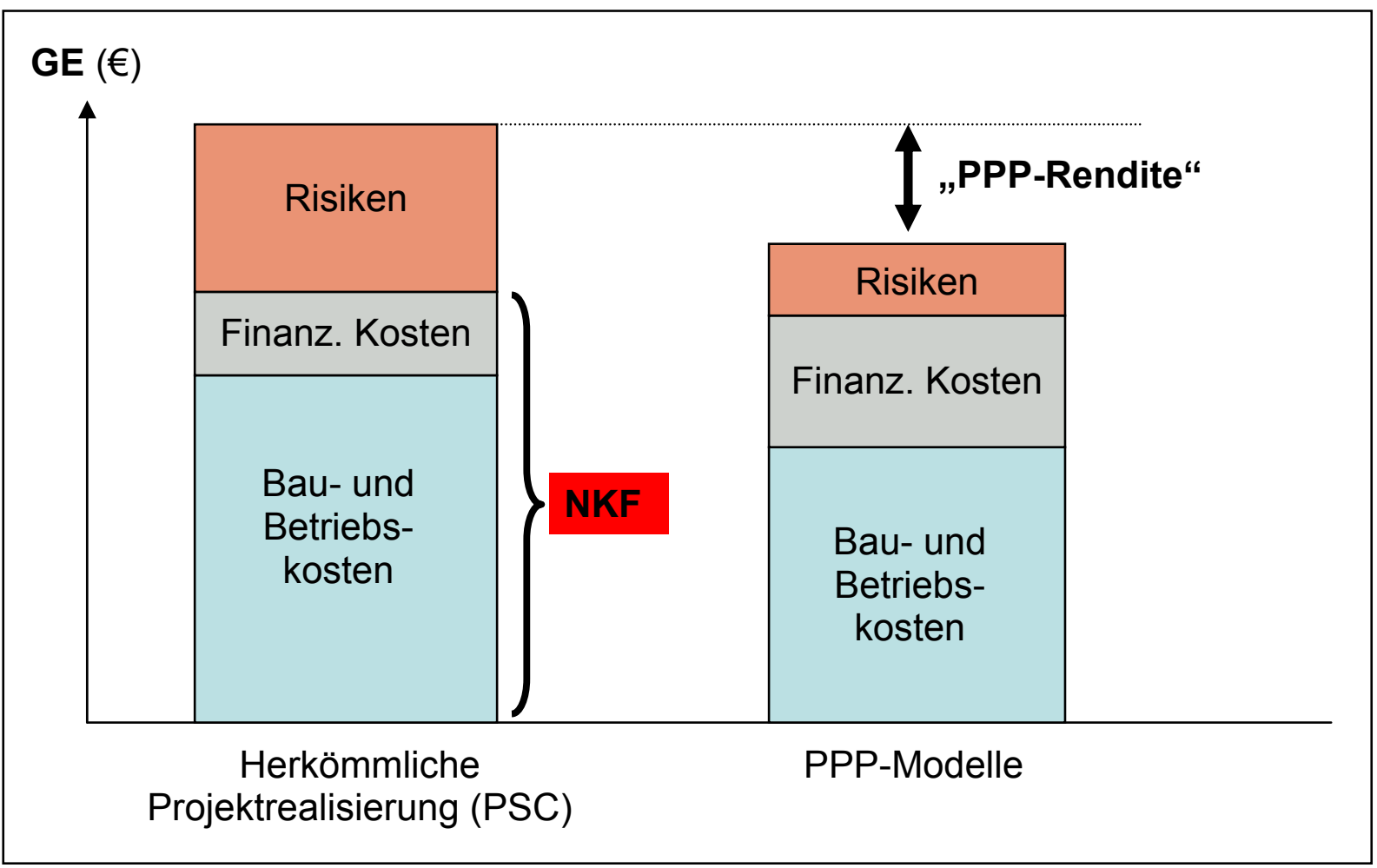

Abb. 2: Öffentliche Kostenstruktur als Vergleichsgrundlage für PPP-Modelle (PSC) Quelle: Vgl. Budäus/Grüb (2006)

Abbildung 2 gibt die unterstellten Ursachen für die Kostenvorteile einer PPP-Lösung an. Die Bau- und Betriebskosten sind geringer als bei einer herkömmlichen Projektrealisierung, die Finanzierungskosten machen dagegen einen höheren Anteil der Kosten aus. Durch die Möglichkeit der Verlagerung von Risiken, durch eine Risikoaufteilung mit dem privaten Partner entsteht eine „PPP-Rendite“. Als Folge wird die PPP als finanziell vorteilhafter im Vergleich zu der herkömmlichen Realisierung eines Projektes durch die öffentliche Hand vermutet.

Trotz der Tatsache, dass PPP ein viel diskutiertes Konzept zur Verwaltungsmodernisierung darstellt und als Ansatz zur Entlastung öffentlicher Haushalte angesehen wird, bestehen eine Reihe offener Fragen und Hemmnisse, die teilweise mit dem jüngst verabschiedeten ÖPP-Beschleunigungsgesetz beseitigt werden sollen. Das ÖPPBeschleunigungsgesetz befasst sich mit den rechtlichen Voraussetzungen von PPP, v.a. im Bereich des Steuer-, Vergabe-, Gebühren-, Investment- und Haushaltsrechts. Es wurden einige institutionelle Rahmenbedingungen festgelegt, die eine einfachere und schnellere Umsetzung von PPP-Projekten gewährleisten sollen. Zudem enthält auch der jüngste Koalitionsvertrag vom 11. November 2005 explizite Hinweise auf weitere Maßnahmen zur Unterstützung von PPP und zum Einsatz privaten Kapitals in Infrastrukturprojekten sowie im Hoch- und Tiefbau. 


\section{Praxisstudie PPP in der Schweiz}

Vor dem Hintergrund dieser Situation und dem Diskussionsstand von PPP-Ansätzen in der Praxis hat Urs Bolz eine neue umfassende Studie zu Public Private Partnership herausgegeben, die zwar primär auf die Situation in der Schweiz ausgerichtet ist, gleichwohl zahlreiche grundsätzliche Überlegungen sowie einen Überblick über praktische PPP-Projekte im europäischen Raum gibt. Entstanden ist die Studie unter Förderung von PricewaterhouseCoopers durch ein Projektteam unter Leitung von Urs Bolz, das auch in unterschiedlicher Zusammensetzung die einzelnen Teile der Studie bearbeitet hat. Ergänzt wurde das Projektteam durch eine Steuergruppe, die sich aus Verwaltungsmitgliedern der Schweiz sowie aus dem Banken-, Bau- und Beraterbereich zusammensetzt. In die Studie sind zudem die Erfahrungen einer Reihe von weiteren Fachleuten eingeflossen, die teilweise in einer eigenständigen Begleitgruppe zu dem Projektteam zusammengefasst waren. Die Studie von Urs Bolz stellt, aufgrund der umfassenden konzeptionellen Grundlagen und zahlreicher Anwendungsbeispiele die aktuell umfassendste Studie im Bereich der praxisorientierten PPP-Literatur dar. ${ }^{6}$ Zielsetzung der Arbeit ist es, Politik, Verwaltung und Wirtschaft in der Schweiz für das reale Phänomen „Public Private Partnership“ zu sensibilisieren sowie ein PPP-Kompetenznetzwerk zu schaffen. Letzteres orientiert sich dabei an den so genannten PPP-Task-Forces auf Bundes- und teilweise auch auf Landesebene in Deutschland. Die „Grundlagenstudie“ wendet sich an die Praxis und versucht alle wesentlichen Aspekte von PPP im internationalen Kontext abzudecken. Zwar wendet sich die Arbeit in erster Linie an die Praxis und stellt keinen Anspruch auf eine Grundlagenstudie im wissenschaftlichen Sinne, gleichwohl liefert sie auch mit ihrer umfassenden und fundierten praktischen Ausrichtung für den wissenschaftlichen Bereich eine Reihe von Anhaltspunkten und zeigt nicht zuletzt eine Vielzahl von zukünftigen Klärungsbedarfen auf, etwa hinsichtlich der Berücksichtung von PPP im reformierten öffentlichen Rechnungswesen.

Besonders hervorzuheben ist zunächst einmal, dass PPP von den Autoren nicht auf PFI eingeengt, sondern wesentlich weiter gefasst wird. PPP stellt einen wesentlichen Baustein der modernen Verwaltung dar. Faktisch wird hiermit auf den Ursprung von PPP in den 40er Jahren des vergangenen Jahrhunderts an der Ostküste in Amerika verwiesen, bei der PPP zur Restrukturierung und ökonomischen Erneuerung der Pittsburgh-Region erstmals eine wesentliche Rolle spielte. Um das Zentrum von Pittsburgh wiederzubeleben bzw. attraktiver zu gestalten, wurde damals die „Allegheny Conference on Community Development" gegründet, eine Form der informellen Zusammenarbeit zwischen der privaten Wirtschaft und der Stadt. Dieses Beispiel verdeutlicht den ursprünglichen PPP-Begriff die öffentliche und private Hand kooperieren auf formellem und/oder informellem Weg, um ein gemeinsames Ziel zu erreichen. ${ }^{7}$

6 Vgl. dazu neben der Beratergruppe - „PPP im öffentlichen Hochbau“ (2003) etwa Bertelsmann Stiftung, Clifford Chance Pünder, Initiative D21 (2003); Jacob/Kochendörfer (2000); Pfeiffer (2004).

7 Vgl. Budäus/Grüning/Steenbock (1997), S. 19 f. 
Konzeptionell orientiert sich die Studie von Urs Bolz an den oben skizzierten beiden Grundkategorien von PPP. Dabei werden die Projekt-PPP primär als Beschaffungs-PPP mit der Zielsetzung einer wirtschaftlicheren öffentlichen Beschaffung interpretiert, während die Organisations-PPP weitgehend als Aufgabenerfüllungs-PPP bezeichnet wird. Inhaltlich ist die Studie in fünf Teilen und einem umfassenden Anhang aufgebaut. Teil I (61 Seiten) setzt sich mit den Grundlagen von PPP auseinander. Hier stehen die Idee, der Begriff von PPP sowie der PPP-Prozess, die Risiken und die unterschiedlichen Wirkungen von PPP im Mittelpunkt. PPP wird als Beschaffungsprozess mit den unterschiedlichen Phasen strukturiert, ein Ansatz, auf den in den folgenden Kapiteln immer wieder konsequent zurückgegriffen wird. Auch findet sich hier eine Abgrenzung von PPP zu den klassischen Formen der Einbeziehung privater Unternehmen in die Erfüllung öffentlicher Aufgaben ebenso wie ein Überblick über verschiedene PPP-Modelle. Bei der Analyse der Wirkungen von PPP geht es sowohl um die Chancen differenziert nach der öffentlichen und privaten Sichtweise als auch um die mit PPP verbundenen Gefahren und Risiken.

Der zweite Teil (59 Seiten) setzt sich mit den PPP-Potenzialen in der Schweiz auseinander und konzentriert sich insbesondere auf die Bereiche Hochbau, Verkehr, Standortentwicklung und -förderung sowie E-Government. Behandelt werden hier auch Ansätze und Maßnahmen zur Förderung von PPP, wobei als zentrales Problem die politische Unterstützung gesehen wird.

Teil III (87 Seiten) befasst sich zum einen mit dem Zusammenhang von PPP und Finanzen. Zum anderen geht es um das Problem der Wirtschaftlichkeitsanalyse und um in diese einbezogene Risikoaspekte. Bezugsfeld sind hier Infrastrukturprojekte. Ganz konkret wird dabei die Wirtschaftlichkeitsanalyse am Praxisbeispiel eines Parkhauses erörtert. Hierbei geht es nicht nur um einen rein betriebswirtschaftlichen Vergleich, sondern auch um die Einbeziehung des „Nutzens im öffentlichen Interesse“. Die beiden Alternativen Erstellung im herkömmlichen Verfahren (Public Sector Comparator) und PPP werden im Rahmen einer Nutzwertanalyse beurteilt, eine Vorgehensweise, die über die häufig geübte Praxis hinausgeht. Anschließend setzen sich die Autoren mit der Risikoanalyse und der Risikoallokation auseinander, wie sie zurzeit in der Praxis diskutiert wird und beziehen auch Überlegungen zum Rating in ihre Untersuchung ein. Aufschlussreich ist auch die Einbeziehung von PPP in die Rechnungslegung öffentlicher Verwaltungen. Hier zeigt sich deutlich, dass sowohl aus Sicht der öffentlichen Rechnungslegung als auch bei den privaten Partnern noch eine Vielzahl von Problemen gelöst werden müssen.

Der vierte Teil (85 Seiten) konzentriert sich auf rechtliche Fragen und hier insbesondere auf Staats- und Verwaltungsrecht, Beschaffungsrecht einschließlich der internationalen Grundlagen und des EU-Vergaberechts, Steuerrecht, Rechtsänderungsrisiken und nicht zuletzt auf ein Vertragscontrolling. Auch hier werden die aktuellen Problemfelder aufgezeigt und Lösungsansätze für die Praxis skizziert.

Der fünfte und letzte Teil (88 Seiten) zeigt die bisherigen Praxiserfahrungen auf internationaler Ebene auf. Dieser Teil basiert auf dem PwC-PPP-Netzwerk. Dabei wird deutlich, dass tendenziell einheitliche Erfahrungen, aber auch Problemfelder in den europäischen Ländern vorliegen. Im Einzelnen wird nach einem Gesamtüberblick über die europäische 
Entwicklung, über den Stand der Diskussion und Projekte in Großbritannien, Deutschland, Niederlande, Frankreich und Österreich berichtet. Dieses Kapitel abschließend greifen die Autoren noch einmal die Situation in der Schweiz auf und dokumentieren die schweizerischen Kooperationsprojekte. Im Anhang werden ergänzend Praxishilfen wie eine PPP-Risikomatrix sowie PPP-spezifische Planerfolgsrechnung, Plankapitalflussrechnung, Planbilanz und Liquiditätsplanung aufgezeigt.

Diese Darstellung ergänzend zeichnet sich ab, dass in Zukunft ganze gesellschaftliche Sektoren durch Kooperationsansätze zwischen Privatwirtschaft und öffentlichem Sektor geprägt sein werden. So gilt dies für den Gesundheitsbereich in Deutschland, der sich zunehmend durch PPP und Privatisierung neu strukturiert. Auch die Konzeption von Kooperationsansätzen zwischen privaten und öffentlichen Institutionen auf dem Gebiet von Wissenschaft und Forschung sind von großem Interesse. Ebenso werden die schon immer bestehenden Kooperationskonzepte im Bereich der Raum- und Stadtentwicklung auch in Zukunft weiterhin für PPP von Bedeutung sein. Schließlich zeichnen sich, wenn auch nicht öffentlich diskutiert, umfassende und weit reichende Kooperationsansätze im Sicherheitsbereich ab. Nicht zuletzt erlangt die Hafenwirtschaft, sowohl in den Niederlanden als auch in Deutschland eine wachsende Bedeutung in der Diskussion um PPP. Trotz der wachsenden Bedeutung wird PPP möglicherweise auch einen Belastungsfaktor für den öffentlichen Sektor darstellen, wie er sich zurzeit in Deutschland bei der Post AG abzeichnet. Die personelle Entlastung nach einer Privatisierung erfolgt hier zu einem nicht unwesentlichen Teil durch eine Verlagerung der Kosten auf den öffentlichen Sektor. Schließlich wird in Zusammenhang mit PPP in Zukunft zu diskutieren sein, inwieweit es sich hierbei um ein neues Instrument der Social Responsibility von Unternehmen handelt und inwieweit es gelingt, über PPP die Potenziale und Ressourcen innerhalb einer Region zu bündeln und zu nutzen.

Insgesamt gibt die Arbeit von Urs Bolz und seinem Projektteam einen guten Überblick über die Grundlagen und Probleme von PPP sowie über die Anforderungen an eine funktions- und leistungsfähige Ausgestaltung dieser Kooperationsansätze. Mittels der Darstellung der Praxisbeispiele in den europäischen Staaten wird ein umfassendes Bild über den aktuellen Entwicklungsstand in Europa einschließlich der bisherigen Erfahrungen gegeben. Zudem zeigen die Autoren die konkreten Entwicklungsmöglichkeiten, Potenziale, aber auch Förderungsbedarfe von PPP in der Schweiz auf. Das Buch überzeugt durch einen klaren Aufbau und eine einheitliche Strukturierung.

\section{Literaturverzeichnis}

Beratergruppe - „PPP im öffentlichen Hochbau“ (2003), Gutachten zur PPP im öffentlichen Hochbau Band I bis IV, Berlin

Bertelsmann Stiftung, Clifford Chance Pünder, Initiative D21 (Hrsg.) (2003), Prozessleitfaden Public Private Partnership, Berlin

Budäus, Dietrich und Birgit Grüb (2006), Public Private Partnership, Berlin (in Vorbereitung)

Budäus, Dietrich und Gernod Grüning (1997), Public Private Partnership - Konzeption und Probleme eines Instruments zur Verwaltungsreform aus Sicht der Public Choice Theorie, in: Public Private Partnership - Neue Formen öffentlicher Aufgabenerfüllung, hrsg. von Dietrich Budäus und Peter Eichhorn, Baden-Baden, S. 25-66 
Budäus, Dietrich, Gernod Grüning und Andreas Steenbock (1997), Public Private Partnership I - State of the Art Hodge, Graeme und Carsten Greve (Hrsg.) (2005), The Challenge of Public Private Partnership - Learning from International Experience, Cheltenham, Northampton

Jacob, Dieter und Bernd Kochendörfer (2000), Private Finanzierung öffentlicher Bauinvestitionen - ein EUVergleich, Berlin

Kommission der Europäischen Gemeinschaften (2004), EU-Grünbuch zu Öffentlich-Privaten Partnerschaften und den gemeinschaftlichen Rechtsvorschriften für öffentliche Aufträge und Konzessionen

Kruzewicz, Michael (1993), Lokale Kooperation in NRW - Public Private Partnership auf kommunaler Ebene, Duisburg

Pfeiffer, Michael (2004), Immobilienwirtschaftliche PPP Modelle im Schulsektor - Großbritannien und Deutschland im Vergleich, Hamburg 